\title{
Condição de saúde bucal da população idosa brasileira: uma revisão integrativa da
}

\section{literatura}

\author{
Oral health of brazilian older population: an integrative literature review \\ Estado de salud bucal de la población anciana brasileña: revisión integradora de la literatura
}

Recebido: 09/11/2021 | Revisado: 19/11/2021 | Aceito: 23/11/2021 | Publicado: 04/12/2021

\author{
Crislane de Lira Silva \\ ORCID: https://orcid.org/0000-0001-9180-3017 \\ Universidade Federal do Amazonas, Brasil \\ E-mail: lirasilva.cris@gmail.com \\ Ana Paula Souza Galvão \\ ORCID: https://orcid.org/0000-0002-2214-8001 \\ Universidade Federal do Amazonas, Brasil \\ E-mail: paulinhagalvao52@gmail.com \\ Yan Nogueira Leite de Freitas \\ ORCID: https://orcid.org/0000-0002-5843-4878 \\ Universidade Federal do Amazonas, Brasil \\ E-mail: yannogueira@ufam.edu.br
}

\begin{abstract}
Resumo
Com o passar do tempo, o corpo humano apresenta um processo de desgaste natural, devido ao envelhecimento. Com a cavidade bucal não é diferente e, infelizmente, o edentulismo ainda representa um dos principais agravos à saúde bucal da população idosa. Objetivo: revisar a literatura científica acerca da condição de saúde bucal das pessoas idosas institucionalizadas e não institucionalizadas brasileiras. Metodologia: trata-se de uma revisão integrativa da literatura, a partir de artigos científicos indexados nas principais bases de dados da área da saúde nos últimos 10 anos. Utilizou-se como palavras-chave: "bucal"; "health"; "older"; "oral"; "condition"; "institutionalized"; "asylu" e descritores: "oral health"; "elderly"; "aged"; "institutionalization". Resultados: após aplicação dos critérios de elegibilidade, dos 2267 artigos recuperados, apenas 8 foram incluídos na revisão. Observou-se que a condição de saúde bucal da população idosa brasileira é precária, com altos índices de CPO-D e perda dentária. A população idosa institucionalizada apresenta, ainda, índices mais elevados se comparado aos indivíduos idosos da comunidade. Conclusão: a revisão em questão confirmou que as pessoas idosas brasileiras apresentam uma condição de saúde bucal preocupante, porém a institucionalização se associa a uma condição de saúde bucal ainda pior, pela vulnerabilidade em que esses indivíduos se encontram. Assim, cabe refletirmos sobre as iniquidades à saúde impostas a essa população e a necessidade de políticas públicas de saúde bucal específicas à população idosa brasileira.
\end{abstract}

Palavras-chave: Idoso; Saúde bucal; Institucionalização.

\begin{abstract}
Over time, the human body undergoes a process of natural wear and tear due to aging. With oral cavity is no different and, unfortunately, edentulism still represents one of the main oral health problems inolder population. Aim: to review the scientific literature about oral health status of institutionalized and non-institutionalized older people in Brazil. Methodology: this is an integrative literature review, based on scientific articles indexed in the main health area databases in the last 10 years. The following keywords were used: "bucal"; "health"; "older"; "oral"; "condition"; "institutionalized"; "asylu" and descriptors: "oral health"; "elderly"; "aged"; "institutionalization". Results: after applying the eligibility criteria, 2267 articles were retrieved, but only 8 were included in the review.It was observed that the brazilian older oral health condition is precarious, with high rates of DMFT and tooth loss.The aged institutionalized population also presents higher rates when compared to aged individuals in the community.Conclusion: the review in question confirmed that older brazilians have a worrying oral health condition, but institutionalization is associated with an even worse oral health condition, due to the vulnerability of these individuals. Thus, it is worth reflecting about health inequities imposed on this population and the need for public oral health policies specific to the aged population in Brazil.
\end{abstract}

Keywords: Aged; Oral health; Institutionalization.

\section{Resumen}

Con el tiempo, el cuerpo humano sufre un proceso de desgaste natural debido al envejecimiento. Con la cavidad bucal no es diferente y, lamentablemente, el edentulismo sigue representando uno de los principales problemas para la salud bucal de la población anciana. Objetivo: revisar la literatura científica sobre la condición de salud bucal de ancianos 
institucionalizados y no institucionalizados en Brasil. Metodología: se trata de una revisión integradora de la literatura, basada en artículos científicos indexados en las principales bases de datos del área de la salud en los últimos 10 años. Se utilizaron las siguientes palabras clave: "bucal"; "health"; "older"; "oral”; "condition"; "institutionalized"; "asylu” y descriptores: "oral health"; "elderly"; "aged”; “institutionalization”. Resultados: después deaplicar los criterios de elegibilidad, de los 2267 artículos recuperados, solo 8 fueron incluidos en la revisión. Se observó que el estado de salud bucal de la población anciana brasileña es precario, con altas tasas de CPOD y pérdida de dientes.La población anciana institucionalizada también presenta tasas más altas en comparación con las personas mayores de la comunidad.Conclusión: la revisión en cuestión confirmó que los ancianos brasileños tienen una condición de salud bucal preocupante, pero la institucionalización se asocia con una condición de salud bucal aún peor, debido a la vulnerabilidaddónde están. Por lo tanto, vale la pena reflexionar sobre las inequidades en salud impuestas a esta población y la necesidad de políticas públicas de salud bucodental específicas para la población anciana en Brasil.

Palabras clave: Anciano; Salud bucal; Institucionalización.

\section{Introdução}

Com o passar do tempo, o corpo humano naturalmente passa pelo processo de envelhecimento, passando por alterações neurobiológicas, químicas e funcionais (Santos et al., 2009). Desse modo, a cavidade bucal sofre mudanças e um dos agravos mais frequentes é o edentulismo, que impacta funcionalmente, emocionalmente e psicologicamente à população idosa (Rosendo et al., 2017). O envelhecimento da população é um acontecimento no mundo todo que representa avanços científicos e tecnológicos (Lucchesi, 2017).

Por muito tempo, o modelo assistencial/mutilador, desassistia a saúde bucal da pessoa idosa, por acreditar que pelos altos índices de edentulismo e uso de prótese, não havia necessidade do acompanhamento odontológico dessa população (Schroeder et al., 2020). Desde 2018, o Ministério da Saúde instituiu o cuidado a atenção odontológica para o idoso no Sistema Único de Saúde, mas na prática ainda é observado o pensamento obsoleto desconsiderando a importância da saúde bucal da pessoa idosa, fazendo com que essa assistência não seja priorizada efetivamente (Brasil, 2018).

No Brasil, aos 60 anos o indivíduo é considerado pessoa idosa e, através do estatuto do idoso, seus direitos são assegurados (Brasil, 2012). Entretanto, ainda não existe uma normatização quanto ao cuidado em saúde bucal da população idosa, sobretudo os institucionalizados, onde podemos notar ausência de cuidados básicos, planejamento para serviços especializados e falta de capacitação dos cuidadores, que muitas vezes não possuem conhecimento sobre as alterações bucais comuns ao envelhecimento. Por outro lado, processos demenciais e dependências funcionais, frequentes nessa população, dificultam o cuidado adequado a esses indivíduos, os tornando ainda mais suscetíveis às doenças e agravos bucais. Portanto, as pessoas idosas institucionalizadas são mais suscetíveis às más condições bucais, por mudanças metabólicas, uso de medicamentos, fatores nutricionais, doenças crônicas, incapacidade funcional e mental (Sales et al. 2017).

Em contrapartida, as pessoas idosas não institucionalizadas, em geral, são mais independentes e possuem maior acesso aos serviços de saúde (Sória et al., 2019). Ainda assim, a saúde bucal desse grupo é preocupante, pois existe uma falta de conhecimento sobre a necessidade de cuidados à saúde bucal nessa faixa etária, prejudicando o uso dos serviços odontológicos (Silva et al., 2016).

Apesar de existirem algumas controvérsias com relação à condição bucal desses dois grupos estudados, a literatura a respeito é vasta, ainda existem muitas questões a serem levantadas, referente ao aumento da expectativa de vida e há questionamentos em relação ao limite biológico e o desenvolvimento de novas tecnologias para tratar incapacidades e doenças crônicas, que são muito comuns nesse período da vida (Kanso, 2013). Dessa forma, o presente trabalho tem como objetivo realizar uma revisão integrativa para avaliar a condição de saúde bucal das pessoas idosas institucionalizadas e não institucionalizadas brasileiras, além de compreender os determinantes envolvidos nesse processo. 


\section{Metodologia}

A fim de responder aos objetivos propostos, optou-se por realizar uma revisão integrativa da literatura. Este tipo de revisão possibilita uma investigação mais ampla do fenômeno estudado, justamente por considerar uma amostra diversificada de estudos, com diferentes abordagens e metodologias. Logo, em geral, a revisão integrativa gera um panorama diversificado e compreensível do objeto de estudo investigado (Souza, et al., 2010).

- Estratégia de busca de dados

Foi realizado um levantamento nas bases de dados PubMed, Scopus, Web of Science, Embase e Lilacs e no portal de buscas Google Acadêmico. Usando as palavras-chave: "bucal"; health"; "older"; “oral”; "condition"; "institutionalized"; "asylu” e descritores: "oral health"; "elderly"; “aged"; "institutionalization”, combinados por operadores booleanos "and", "or" e "and not" (Quadros 1 e 2). A coleta de dados foi conduzida por uma pesquisadora calibrada para evitar possíveis vieses, calculou-se a estatística kappa intraexaminador $(0,856 ; \mathrm{p}<0,001)$, considerando duas buscas na mesma base de dados no intervalo de um mês.

Quadro 1: Estratégia de busca para os artigos sobre a saúde bucal da pessoa idosa não institucionalizada no estudo intitulado "Saúde Bucal da Pessoa Idosa Brasileira". Manaus/AM, 2021.

\begin{tabular}{|c|c|c|}
\hline Base de dados & Detalhes da estrategia & Resultados \\
\hline Pubmed/Medline & $\begin{array}{l}\text { ("aged" OR "elderly" OR "older") ("oral health" OR "bucal health" OR "oral condition") } \\
\text { "Brazil" }\end{array}$ & 476 \\
\hline Web of Science & $\begin{array}{c}\text { (("aged" OR "elderly" OR "older") ("oral health" OR "bucal health" OR "oral condition") } \\
\text { "Brazil"" NOT "child*" NOT "adolescent"*) }\end{array}$ & 354 \\
\hline Scopus & $\begin{array}{l}\text { ("aged" OR "elderly" OR "older") AND ("oral health" OR "bucal health" OR "oral } \\
\text { condition") AND "Brazil"" }\end{array}$ & 197 \\
\hline Embase & $\begin{array}{l}\text { ("aged" OR "elderly" OR "older") AND ("oral health" OR "bucal health" OR "oral } \\
\text { condition") AND "brazil" NOT "child"" NOT "adolescent"" }\end{array}$ & 195 \\
\hline Lilacs & $\begin{array}{c}\text { ("oral health" or "bucal health") (aged or older or elderly) brazil and not child" and not } \\
\text { adolescent and not institutionalized }\end{array}$ & 205 \\
\hline Google acadêmico & "saude bucal" "idoso" "brasil" "dissertaçâo" "tese" "tcc" "livro" & 367 \\
\hline
\end{tabular}

Fonte: Autores.

Quadro 2: Estratégia de busca para os artigos sobre a saúde bucal da pessoa idosa institucionalizada no estudo intitulado

\begin{tabular}{|c|c|c|}
\hline Base de dados & Detalhes'da estratégiaa & Resultados $\square$ \\
\hline Pubmed/Medliner & $\begin{array}{l}\text { ("oral health".OR-"bucal health") ("aged".OR-"elderly".OR-"older"). } \\
\text { ("institutionalized". OR,"institutionalization"),"Brazil"*a }\end{array}$ & 140 \\
\hline Web-of-Science & 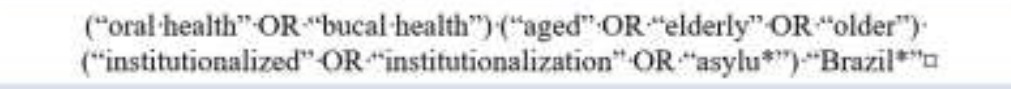 & 210 \\
\hline Scopusa & 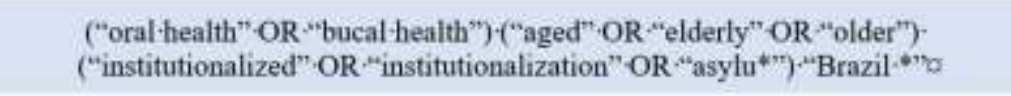 & $200 \mathrm{a}$ \\
\hline Embase $\square$ & 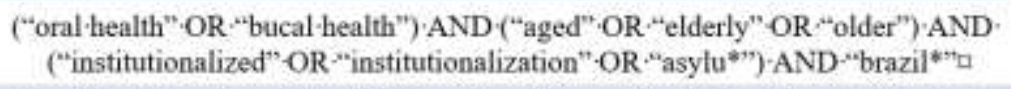 & 290 \\
\hline Lilacsa & $\begin{array}{l}\text { ("oral health" or-"bucal health")(aged or older or elderly) brazil institutionalized } \\
\text { and not-child"-and not-adolescent" }\end{array}$ & 220 \\
\hline Google acadêmicon & "saúde bucal" "idoso" "brasil" "dissertação" "tese" tcc" "livro" & $367 \square$ \\
\hline
\end{tabular}


- Critérios de elegibilidade dos artigos

Foram inclusos estudos completos indexados entre 2011 e 2021, nos idiomas inglês, português e espanhol e que respondiam a uma das perguntas de pesquisa: “Qual a condição de saúde bucal da pessoa idosa institucionalizada?” ou “Qual a condição de saúde bucal da pessoa idosa não-institucionalizada?”, aqueles que se baseavam em índices ou indicadores validados, que apresentavam qualidade metodológica, garantindo a evidência dos achados e avaliando o desfecho no contexto brasileiro.

- Processo de seleção dos artigos

O processo de seleção dos artigos, primeiramente, foi realizado através da leitura dos títulos e resumos dos estudos recuperados. Foram lidos integramente aqueles em que os títulos e resumos responderam aos critérios propostos. Foram excluídos os estudos duplicados, aqueles sem acesso completo, os que não caracterizavam a saúde bucal de forma clara e objetiva de acordo com os principais índices de saúde bucal. Dessa forma, as informações coletadas dos artigos selecionados foram organizadas em um quadro contendo: título da publicação, autores, ano de publicação, idioma publicado, país, amostra, condições de saúde bucal e contexto de vida.

\section{Resultados e Discussão}

Foram identificadas 2267 referências nas bases de dados pesquisadas. A seleção por título resultou em 473 artigos para pessoas idosas institucionalizadas. Desses, 80 resumos foram avaliados e 4 artigos foram incluídos. Para pessoas idosas não institucionalizadas, foram encontrados 1794 artigos e 80 resumos foram avaliados, dos quais 4 artigos foram incluídos. Os fluxogramas contidos nas (Figura 1 e 2) apresentam o processo de seleção dos artigos. Dos 8 artigos selecionados, 2 (25 \%) estavam disponíveis na língua inglesa e $6(75 \%)$ na língua portuguesa, todos os estudos foram conduzidos no Brasil e publicados entre 2011 e 2021. 
Figura 1: Fluxograma de seleção de artigos incluídos na revisão sobre saúde bucal de pessoas idosas institucionalizadas no Brasil. Manaus/AM, 2021.

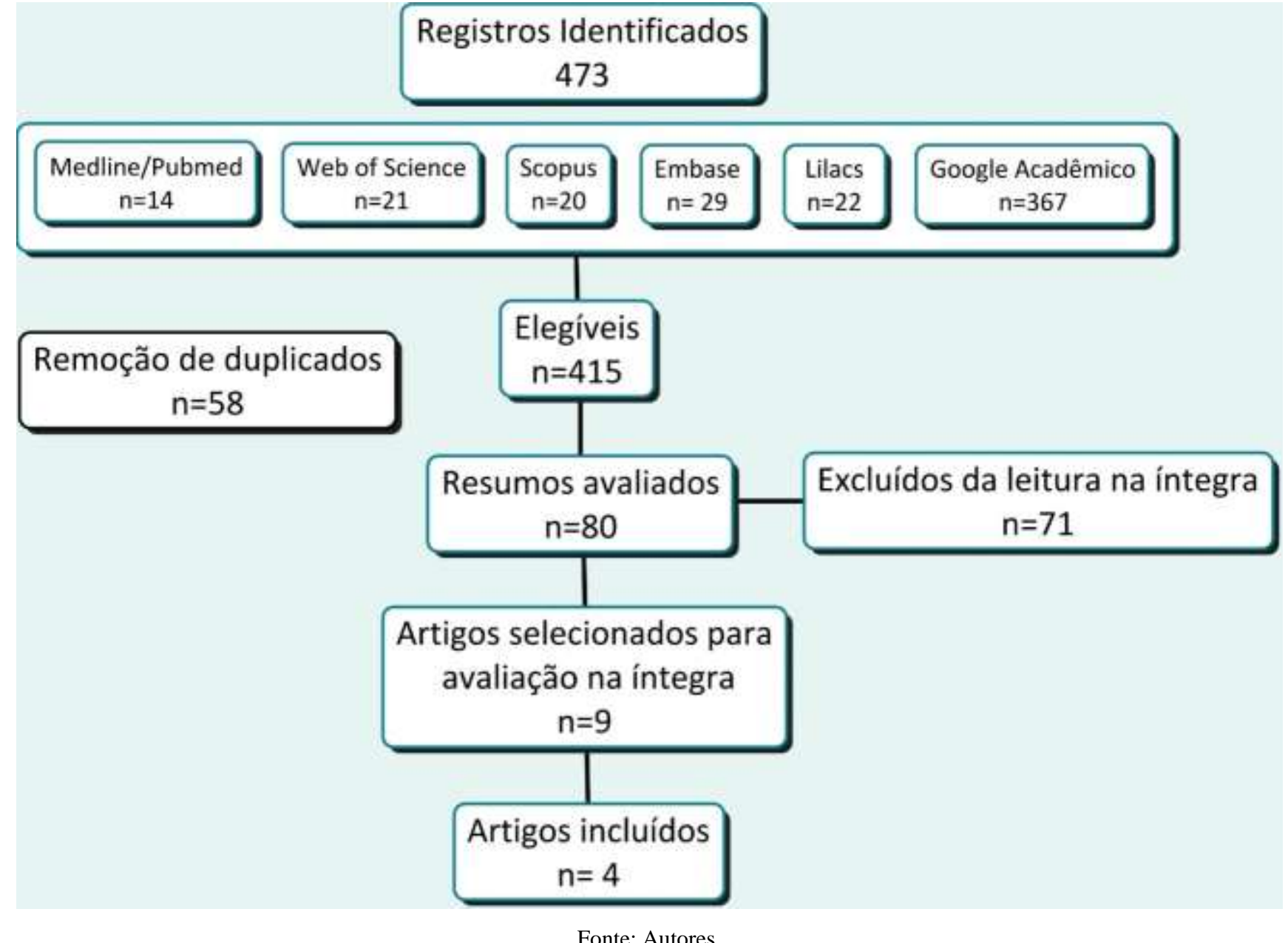


Figura 2: Fluxograma de seleção de artigos incluídos na revisão sobre saúde bucal de pessoas idosas não institucionalizadas no Brasil. Manaus/AM, 2021.

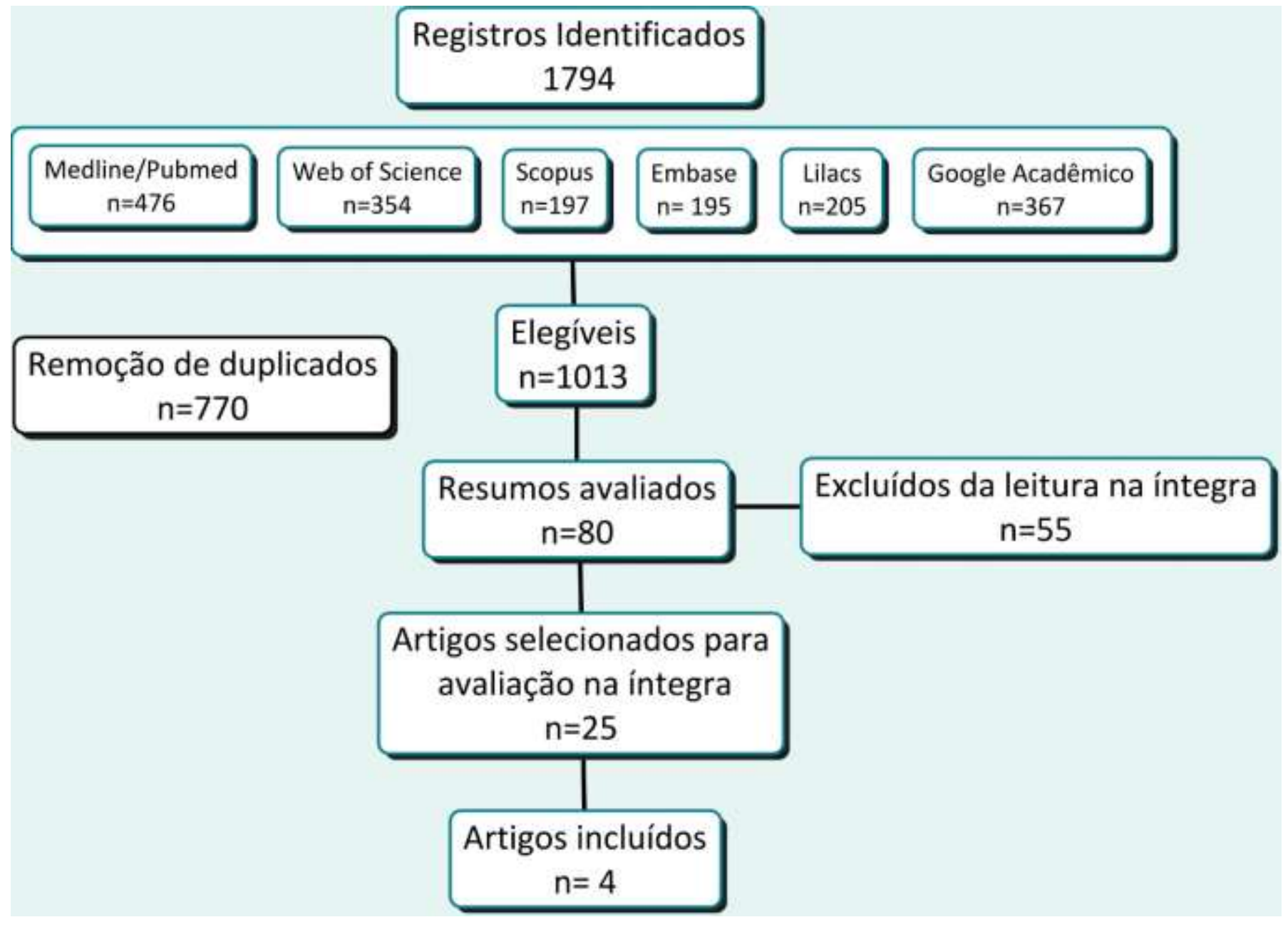

Fonte: Autores.

Quanto às amostras estudadas, o número de indivíduos variou de 55 a 1030. Dentre os idosos institucionalizados, observou-se alta média do índice CPO-D, variando de 30,4 a 38,0, alta prevalência de edentulismo e, portanto, alto uso e necessidade de próteses dentárias. Para os não-institucionalizados, a situação foi bem semelhante. No entanto, observou-se uma média do índice CPO-D um pouco menor, variando de 27,6 a 28,7 e, assim, menor prevalência para as demais variáveis observadas, conforme demonstram os quadros 3 e 4.

Quanto aos estudos que avaliaram a condição de saúde bucal da população idosa no sul do Brasil, Beloti et al. (2011), verificaram em um estudo com 82 pessoas idosas residentes em duas Instituição de Longa Permanência (ILPI), que a prevalência de edentulismo foi de 57,3\% e, desses, 40,5\% usavam prótese há mais de dez anos. Verificou-se que 73,2\% apresentaram necessidade de reabilitação no arco superior com prótese total e 67,1\% no arco inferior, independente de já serem portadores de próteses ou não.

Kurihara et al. (2013), por sua vez, apresentaram dados comparativos entre pessoas idosas institucionalizadas e não institucionalizadas, com 86 pessoas idosas, sendo 38 institucionalizadas e 48 não institucionalizados, onde o índice de CPO-D foi 38 e mostrou que houve um número elevado de dentes restaurados para pessoas idosas institucionalizadas. Para este grupo, ainda, observou-se que houve mais uso de próteses dentárias $(\mathrm{p}=0,01)$. 
Quadro 3: Saúde bucal de pessoas idosas institucionalizadas no Brasil. Manaus/AM, 2021.

\begin{tabular}{|c|c|c|c|}
\hline Autor/Ano & População & Limitações & Resultados \\
\hline $\begin{array}{l}\text { Beloti, A. M., et al. } \\
\text { (2011) }\end{array}$ & $\begin{array}{l}82 \text { pessoas } \\
\text { idosas. }\end{array}$ & $\begin{array}{l}\text { Ausência do cálculo do } \\
\text { tamanho da amostra }\end{array}$ & $\begin{array}{c}\text { Edentulismo: } 57,3 \% \\
\text { Usavam prótese: } 57,3 \% \\
\text { Necessidade de prótese superior: } 73,2 \% \\
\text { Necessidade protéticainferior: } 67,1 \% \text {. }\end{array}$ \\
\hline $\begin{array}{l}\text { Ribeiro, I. L. A. } \\
\text { (2012) }\end{array}$ & $\begin{array}{l}55 \text { pessoas } \\
\text { idosas }\end{array}$ & $\begin{array}{l}\text { Ausência do cálculo do } \\
\text { tamanho da amostra e } \\
\text { calibração dos } \\
\text { pesquisadores. }\end{array}$ & $\begin{array}{c}\text { Cariados com necessidade de restauração: } 4,9 \% \text {; Cariadoscom } \\
\text { indicação de exodontia: } 1,6 \% ; \\
\text { Dente restaurado: } 0,8 \% ; \\
\text { Edentulismo parcial: } 36,4 \% ; \\
\text { Edentulismo total: } 63,6 \% ; \\
\text { Prótese total removível: } 40 \% ; \\
\text { Prótese parcial removível: } 9,1 \% \text {. }\end{array}$ \\
\hline $\begin{array}{l}\text { Sá, I. P. C., et al. } \\
\text { (2012) }\end{array}$ & $\begin{array}{l}58 \text { pessoas } \\
\text { Idosas. }\end{array}$ & $\begin{array}{l}\text { Ausência do cálculo do } \\
\text { tamanho da amostra e } \\
\text { calibração dos } \\
\text { pesquisadores. }\end{array}$ & $\begin{array}{l}\text { CPO-D: } 30,4 \\
\text { Faziam uso de prótese dentária: } 55,2 \% \text {; } \\
\text { Não faziam uso de prótese: } 44,9 \% \text {. }\end{array}$ \\
\hline $\begin{array}{l}\text { Kurihara, E., et al. } \\
\qquad(2013)\end{array}$ & $\begin{array}{l}86 \text { pessoas } \\
\text { idosas. }\end{array}$ & $\begin{array}{c}\text { Amostra } \\
\text { não representativa }\end{array}$ & $\begin{array}{l}\text { CPO-D:38 } \\
\text { Faziam uso de prótese dentária: } 81,5 \% \\
\text { Necessitavam de próteses: } 71 \%\end{array}$ \\
\hline
\end{tabular}

Fonte: Autores.

Quadro 4: Saúde bucal de pessoas idosas não institucionalizadas no Brasil. Manaus/AM, 2021.

\begin{tabular}{|c|c|c|c|}
\hline Autor/Ano & População & Limitações & Resultados \\
\hline $\begin{array}{l}\text { Alcântara, C. M.,et al. } \\
\text { (2011) }\end{array}$ & $\begin{array}{l}505 \text { pessoas } \\
\text { idosas. }\end{array}$ & $\begin{array}{l}\text { Autores não apresentaram } \\
\text { limitações. }\end{array}$ & $\begin{array}{c}\text { CPO-D: } 27,6 \text { sendo, } 394 \text { dentes cariados, } 11.863 \\
\text { perdidos e } 1.714 \text { obturados; } \\
\text { Prótese total removível: } 46,9 \% ; \\
\text { Não utilizavam prótese: } 44,2 \% ; \\
\text { Necessitavam de prótese total removível: } 23,8 \% \text {. }\end{array}$ \\
\hline $\begin{array}{l}\text { Schroeder, F. M. M., et al. } \\
\qquad(2020)\end{array}$ & $\begin{array}{l}1.030 \text { pessoas } \\
\text { idosas. }\end{array}$ & $\begin{array}{c}\text { Ausência de calibração dos } \\
\text { pesquisadores. }\end{array}$ & $\begin{array}{c}\text { Uso de algum tipo de prótese dentária: } 74,8 \% ; \\
\text { Edêntulos totais: } 49,9 \% \text {. } \\
\text { Necessitavam de prótese: } 61,8 \% \\
\text { Possuíam até oito dentes em ambas arcadas: } 73 \%\end{array}$ \\
\hline $\begin{array}{l}\text { de Oliveira, T. C., et al. } \\
\text { (2013) }\end{array}$ & $\begin{array}{l}441 \text { pessoas } \\
\text { idosas }\end{array}$ & $\begin{array}{l}\text { Apesar de representativa, } \\
\text { amostra homogênea, } \\
\text { dificultando validade externa. }\end{array}$ & $\begin{array}{c}\text { CPO-D: } 28,7 ; \\
\text { Edêntulos totais: } 50,8 \% \\
\text { Edêntulos funcionais: } 92,7 \% \text {; } \\
\text { Necessidade de prótese superior: } 80 \% \text {; } \\
\text { Necessidade de prótese inferior } 86,4 \% \text {. }\end{array}$ \\
\hline $\begin{array}{l}\text { Silva, A. E. R.,et al. } \\
\text { (2018) }\end{array}$ & $\begin{array}{l}438 \text { pessoas } \\
\text { idosas. }\end{array}$ & $\begin{array}{l}\text { Delineamento do estudo, que } \\
\text { impossibilita estabelecer } \\
\text { relações de causa e efeito entre } \\
\text { a exposição e o desfecho. }\end{array}$ & $\begin{array}{c}\text { Utilizavam algum tipo de prótese dentária: } 85 \% \\
\text { Necessitavam de alguma prótese dentária: } 51 \% \\
\text { Edêntulos: } 51 \% \text {. }\end{array}$ \\
\hline
\end{tabular}

Fonte: Autores.

Outros dois estudos apresentaram dados de pessoas idosas não institucionalizadas na região sul do país, Schroeder et al. (2020), trouxeram em seu estudo com 1030 pessoas idosas, que em relação aos serviços odontológicos, 86,1\% não os utilizaram. Metade dos indivíduos eram edêntulos totais $(49,9 \%)$ e utilizavam prótese dentária (74,8\%). Em um estudo com 438 pessoas idosas, Silva et al. (2018) estudaram indivíduos com idade entre 60-69 anos e em relação ao serviço odontológico, observou-se que 92,2\% não buscaram regularmente os serviços, 51,0\% necessitavam de alguma prótese dentária, 85,0\% já utilizavam algum tipo de prótese dentária e 51,0\% da amostra investigada nesse estudo era edêntula. É perceptível que os valores mais elevados de edentulismo e necessidade protética são dos indivíduos que moram em ILPI, esta condição pode ser resultado da carência de programas de saúde administrados a esses indivíduos como também pela falta de conhecimento das pessoas responsáveis por essas instituições e pelas pessoas idosas, pois elas parecem não constituírem prioridade nos serviços públicos, mesmo perante os problemas (Beloti et al., 2011).

Na região sudeste do país, Sá et al. (2012) realizaram um estudo com 58 pessoas idosas institucionalizadas e 
constataram um CPO-D de 30,4, sendo 1707 dentes perdidos. Sobre o uso de prótese dentária, 55,2\% faziam uso dessa. Sobre a necessidade de tratamento odontológico, 84,5\% necessitavam de algum tipo de tratamento, sendo os tratamentos mais necessários: exodontia e próteses dentárias.

Um estudo realizado em 2011, por Alcântara e colaboradores, investigaram 505 pessoas idosas não institucionalizadas, revelando 394 dentes cariados, 11.863 perdidos e 1.714 obturados, tendo como resultado um CPO-D de 27,6. Em relação ao uso e necessidade de prótese, 44,2\% não utilizavam próteses, 23,8\% necessitavam de prótese total removível, 46,9\% utilizavam prótese total removível e 54,1\% não apresentavam necessidade de uso de prótese. Pessoas idosas institucionalizadas apresentaram parcelas mais elevadas de cárie, edentulismo e necessidades de próteses quando comparadas aos domiciliados, essas pessoas geralmente apresentam condição bucal diferente da população idosa geral, por conta de diversas situações como, doenças sistêmicas, alterações fisiológicas que o corpo sofre naturalmente e que refletem nos precários cuidados com a saúde bucal, que podem estar, também, relacionadas às limitações motoras, problemas visuais, baixa autoestima e falta de cuidados (Silva et al., 2015).

Uma pesquisa de Ribeiro (2012), com 55 pessoas idosas institucionalizadas, no nordeste brasileiro, obteve os seguintes resultados, em relação aos elementos dentários: 4,9\% estavam cariados, porém com possibilidade de serem restaurados, 1,6\% estavam cariados e necessitavam de exodontias e 0,8\% estavam restaurados. Eram edêntulos parcial 36,4\% e 63,6\% edêntulos totais. Cerca de $80 \%$ dessa população necessitava de prótese dentária e $40 \%$ já fazia uso de prótese total. Em outro estado do nordeste brasileiro, em estudo conduzido por De Oliveira et al. (2013), 441 pessoas idosas não institucionalizadas apresentaram o valor médio do CPO-D de 28,16, com perda dentária correspondendo a 94,1\% desse índice. Em $80 \%$ dos participantes da pesquisa havia a necessidade de prótese superior e 86,4\% de prótese inferior. Eram edêntulos totais $50,8 \%$ e $92,7 \%$ edêntulos funcionais.

Os dados revelaram que o edentulismo ainda é um dos grandes problemas dessa população, além da necessidade de próteses, doenças periodontais, cáries e lesões em tecidos moles. Diante dessa realidade, vale ressaltar que pessoas idosas que vivem em suas casas, com sua família ou cuidadores particulares e que são independentes funcionalmente mostram menos intervenção das comorbidades, que são mais facilmente encontradas naquelas ditas institucionalizadas, apresentando, desse modo, total ligação com uma saúde bucal menos precária, pelo fato dessas pessoas apresentarem maior grau de independência para realizar suas atividades diárias, por exemplo, (Sá et al., 2012).

Cabe, ainda, destacar um estudo de Peres et al. (2019), onde os autores discutem a atual tendência de diminuição da perda dentária em pessoas idosas ao redor do mundo, sobretudo em países em desenvolvimento, como é o caso do Brasil. Tendência que poderá ser observada no próximo levantamento nacional acerca das condições de saúde bucal da população brasileira, o Saúde Bucal (SB) Brasil 2020 (SB BRASIL) que será realizado em 2022. No entanto, esses e outros autores destacam que a diminuição da perda dentária não tem ocasionado uma melhora das condições de saúde bucal desses indivíduos, pois o uso e acesso aos serviços odontológicos continuam insuficientes. Na verdade, o que se têm observado, conforme esses autores e os achados dessa revisão, é que as pessoas idosas estão conseguindo manter por mais tempo os dentes em boca, mas em péssimas condições, acarretando em prejuízos à saúde e funcionalidade dos mesmos (Visscher et al., 2014; Silva, 2016).

Por fim, apesar das contribuições desse estudo no que diz respeito ao conhecimento das condições de saúde bucal das pessoas idosas brasileiras, sabe-se que estudos do tipo revisão integrativa apresentam algumas limitações quanto à evidência dos achados. Reunir estudos com delineamentos, amostras e cenários heterogêneos limitam, em parte, as comparações das condições de saúde bucal entre as pessoas idosas institucionalizadas e não-institucionalizadas. Apesar disso, entendemos que esse tipo de revisão nos permite incluir um número maior de estudos, possibilitando ampliar nosso conhecimento acerca das condições de saúde bucal desses indivíduos em diferentes contextos. Assim, os achados ora apresentados e discutidos, 
certamente, confirmam o que boa parte da literatura traz acerca da saúde bucal dessa parcela da população, confirmando, mais uma vez, a necessidade de políticas públicas e maior atenção à prevenção, promoção e proteção à saúde bucal e geral da pessoa idosa brasileira.

\section{Conclusão}

A revisão em questão confirmou que as pessoas idosas brasileiras apresentam uma condição de saúde bucal preocupante. Foi possível, também, observar que a institucionalização se associa a uma condição de saúde bucal ainda pior, pela vulnerabilidade em que esses indivíduos se encontram. As ILPI, em sua maioria, não conseguem oferecer a esses indivíduos um cuidado à saúde ideal, seja pela falta de recursos e investimentos ou devido à negligência quanto à qualidade de vida dessa população.

Assim, cabe refletirmos acerca das iniquidades em saúde postas a essa população. Demonstra-se que o uso e acesso a serviços odontológicos pelas pessoas idosas brasileiras são precários e, ainda, norteados por práticas mutiladoras. A alta necessidade de próteses dentárias reflete a baixa cobertura dos serviços especializados e a necessidade de políticas públicas de saúde bucal voltadas a essa população. Sabe-se que a saúde bucal é essencial para a socialização e um envelhecimento ativo e saudável.

\section{Referências}

Alcântara, C. M., Dias, C. A., Rodrigues, S. M., \& Reis, F. A. D. (2011). Estudo comparativo da condição de saúde bucal de idosos não institucionalizados de Governador Valadares-MG, com a meta proposta pela Organização Mundial de Saúde para 2010. Physis: Revista de Saúde Coletiva, $21,1023-1044$.

Beloti, A. M., Schwab, B. L., Bertipaglia, T., Nishimori, L. E., \& Fernandes, C. A. M. (2011). Avaliação das condições de saúde bucal de idosos institucionalizados em asilos públicos de Maringá-PR. Ciência, Cuidado e Saúde, 10(1), 096-100.

Brasil. Ministério da Saúde. Secretaria de Atenção à Saúde. Secretaria de Vigilância em Saúde. SB Brasil 2010: Pesquisa nacional de saúde bucal: resultados principais. Brasília: Ministério da Saúde, 2012.

Brasil. Ministério da Saúde. Secretaria de Atenção à Saúde. Departamento de Atenção Básica. A saúde bucal no Sistema Único de Saúde -Ministério da Saúde, 2018.

de Oliveira, T. C., da Silva, D. A., de Freitas, Y. N. L., da Silva, R. L., de Castro Pegado, C. P., \& de Lima, K. C. (2013). Socio-demographic factors and oral health conditions in the elderly: a population-based study. Archiveofgerontologyandgeriatrics, 57(3), 389-397.

Estatuto do idoso: lei federal n ${ }^{\circ} 10.741$, de 01 de outubro de 2003. Brasília, DF: Secretaria Especial dos Direitos Humanos, 2004

Kanso, S. (2013). Processo de envelhecimento populacional: um panorama mundial. In VI Workshop de análise ergonômica do trabalho, III Encontro Mineiro de Estudos em Ergonomia e WIII Simpósio de Programa de Educação Tutorial em Economia Doméstica, Belo Horizonte.

Kurihara, E., Neves, V. J. D., Kitayama, V. S., Endo, M. S., Terada, R. S. S., \& Marcondes, F. K. (2013). Relationship between oral health and psychological factors in institutionalized and non-institutionalized elderly individuals. RGO. Revista Gaúcha de Odontologia (Online), 61(2), 177-186.

Lucchesi, G. (2017). Envelhecimento populacional: perspectivas para o SUS. Brasil 2050: Desafios De Uma Nação Que Envelhece, 43.

Peres, M. A., Macpherson, L. M, Weyant, R. J., Daly, B., Venturelli, R., Mathur, M. R., \& Watt, R. G (2019). Doenças bucais: um desafio global para a saúde pública. The Lancet, 394 (10194), 249-260.

Ribeiro, I. L. A., Veloso, H. H. P., \& de Souza, K. C. (2012). Caracterização da saúde bucal de idosos em uma instituição beneficente de longa permanência de João Pessoa-PB, Brasil. Revista Cubana de Estomatología, 49(3), 193-203.

Rosendo, R. A., Sousa, J. N. L., Abrantes, J. G. S., Cavalcante, A. B. P., \& Ferreira, A. K. T. F. (2017). Autopercepção de saúde bucal e seu impacto na qualidade de vida em idosos: uma revisão de literatura. Revista Saúde \& Ciência Online, 6(1), 89-102.

Sá, I. P. C., Almeida Júnior, L. R. D., Corvino, M. P. F., \& Sá, S. P. C. (2012). Condições de saúde bucal de idosos da instituição de longa permanência Lar Samaritano no município de São Gonçalo-RJ. Ciência \& Saúde Coletiva, 17, 1259-1265.

Sales, M. V. G., Fernandes Neto, J. D. A., \& Catão, M. H. C. V. (2017). Condições de saúde bucal do idoso no Brasil: uma revisão de literatura. Arch Health Invest, 6(3), 120-4.

Santos, F. H. D., Andrade, V. M., \& Bueno, O. F. A. (2009). Envelhecimento: um processo multifatorial. Psicologia em estudo, $14,3-10$. 
Research, Society and Development, v. 10, n. 16, e12101623023, 2021

(CC BY 4.0) | ISSN 2525-3409 | DOI: http://dx.doi.org/10.33448/rsd-v10i16.23023

Schroeder, F. M. M., Mendoza-Sassi, R. A., \& Meucci, R. D. (2020). Condição de saúde bucal e utilização de serviços odontológicos entre idosos em área rural no sul do Brasil. Ciência \& Saúde Coletiva, 25, 2093-2102.

Silva, A. E. R., Echeverria, M. S., Custódio, N. B., Cascaes, A. M., Camargo, M. B. J. D., \& Langlois, C. D. O. (2018). Uso regular de serviços odontológicos e perda dentária entre idosos. Ciência \& Saúde Coletiva, 23, 4269-4276.

Silva, B. L. A., Bonini, J. A., \& Bringel, F. D. A. (2015). Condição de saúde bucal de idosos institucionalizados em Araguaína/TO. Braz J Periodontol, 25(1), 7-13.

Silva, D. A. D., Freitas, Y. N. L. D., Oliveira, T. C. D., Silva, R. L. D., Pegado, C. P. D. C., \& Lima, K. C. D. (2016). Oral health conditions and activities of daily living in an elderly population in Brazil. Revista Brasileira de Geriatria e Gerontologia, 19, 917-929.

Sória, G. S., Nunes, B. P., Bavaresco, C. S., Vieira, L. S., \& Facchini, L. A. (2019). Acesso e utilização dos serviços de saúde bucal por idosos de Pelotas, Rio Grande do Sul, Brasil. Cadernos de Saúde Pública, 35

Souza, M. T. D., Silva, M. D. D., \& Carvalho, R. D. (2010). Revisão integrativa: o que é e como fazer. Einstein (São Paulo), 8, $102-106$.

Visscher, C. M., Lobbezoo, F., \& Schuller, A. A. (2014). Dental status and oral health-related quality of life. A population-based study. Journal of oral rehabilitation, 41(6),416-422. 